\title{
Metabolic profiling in Prader-Willi syndrome and nonsyndromic obesity: sex differences and the role of growth hormone
}

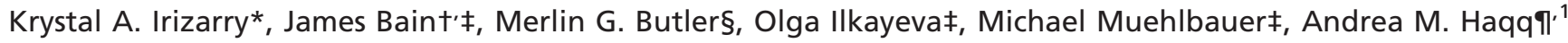 \\ and Michael Freemark*' $\neq^{\prime 1}$ \\ ${ }^{\star}$ Division of Pediatric Endocrinology and Diabetes, Duke University Medical Center, $\dagger$ Division of Endocrinology, Metabolism, and \\ Nutrition, Duke University Medical Center, $\$$ Sarah W. Stedman Nutrition and Metabolism Center and the Duke Molecular \\ Physiology Institute, Duke University, Durham, NC, USA, §Departments of Pediatrics, Psychiatry and Behavioral Sciences, Kansas \\ University Medical Center, Kansas City, KS, USA and 9 Department of Pediatrics, University of Alberta, Edmonton, AB,Canada
}

\section{Summary}

Objectives To identify metabolic factors controlling appetite and insulin sensitivity in PWS and assess effects of GH treatment.

Methods We compared amino acids, fatty acids and acylcarnitines in GH-treated and untreated PWS children and obese and lean controls to identify biomarkers associated with ghrelin, peptide YY and markers of insulin sensitivity (adiponectin and HOMA-IR).

Results Compared with obese controls (OC), children with PWS had fasting hyperghrelinaemia, hyperadiponectinaemia, hypoinsulinaemia and increased ghrelin/PYY. Hyperghrelinaemia, hyperadiponectinaemia and hypoinsulinaemia were more striking in PWS females than males, and decreases in BCAA were detected only in PWS females. GH-treated PWS subjects had lower leptin and higher IGF-1 and adiponectin than untreated subjects; fasting ghrelin, PYY and insulin levels were comparable. Ghrelin correlated inversely with BCAA in PWS but not OC. Adiponectin correlated negatively with BMIz and HOMA-IR in PWS; in contrast, adiponectin correlated more strongly with BCAA than BMIz or HOMA-IR in OC.

Conclusions BCAA levels were lower in PWS females than OC females and correlated inversely with ghrelin. Low BCAA in PWS females may promote hyperghrelinaemia and hyperphagia,

Correspondence: Krystal A. Irizarry, Division of Pediatric Endocrinology and Diabetes, Duke University Medical Center, Lenox Baker Children's Hospital, 3000 Erwin Rd, Suite 200 Box 102820, Durham, NC 27705. Tel.: 919-684-8250; E-mail: Krystal.Irizarry@duke.edu.

and

Andrea M. Haqq, Division of Pediatric Endocrinology, Department of Pediatrics, University of Alberta, 1C4 Walter C. Mackenzie Health Sciences Centre, 8440 - 112 Street NW, Edmonton, AB T6G 2R7. Tel.: 780248-5490; E-mail: andrea.haqq@albertahealthservices.ca and

Michael Freemark, Pediatric Endocrinology and Diabetes, Duke University Medical Center, 3000 Erwin Rd, Suite 200 Box 102820, Durham, NC 27705. Tel.: 919-684-8350; E-mail: Michael.Freemark@duke.edu

${ }^{1}$ co-senior authors. while hyperadiponectinaemia may maintain insulin sensitivity despite excess weight gain. GH treatment may reduce leptin and increase adiponectin, but does not affect fasting ghrelin or PYY.

(Received 13 January 2015; returned for revision 21 January 2015; finally revised 12 February 2015; accepted 26 February 2015)

\section{Introduction}

Prader-Willi syndrome (PWS) is the most common genetic obesity disorder and results from lack of gene expression on paternally inherited chromosome $15 \mathrm{q} 11 \cdot 2-\mathrm{q} 13 .^{1,2}$ The hallmark features of PWS include neonatal hypotonia and failure to thrive in infancy followed by hyperphagia and obesity in early childhood. ${ }^{3}$ This makes PWS a unique model for the study of appetite regulation. In addition to hyperphagia, patients with PWS have hypogonadism and short stature that are theorized to result from hypothalamic dysfunction. ${ }^{3}$ However, the factors regulating appetite and satiety in PWS are poorly understood.

Relative to age- and BMI-matched obese controls, children and adults with PWS have elevated fasting levels of ghrelin, a hormone synthesized primarily by the enteroendocrine cells of the stomach. Ghrelin circulates as acylated and desacylated forms; limited evidence suggests increases in both the acyl and desacyl forms of the hormone in children and adults with PWS. ${ }^{4,5}$ Acylated ghrelin promotes food intake in humans ${ }^{6}$ and fat deposition and weight gain in experimental animals. ${ }^{7}$ The orexigenic effects of ghrelin may be opposed by peptide YY, an anorexigenic hormone secreted by the $\mathrm{L}$ cells of the gastrointestinal tract in response to feeding. ${ }^{8}$ Total and acyl ghrelin levels in healthy subjects fall after food intake and weight gain and rise after weight loss'; the hyperghrelinaemia of overweight PWS patients therefore seems paradoxical. Through binding to the pituitary growth hormone secretagogue receptor (GHS-R), acyl ghrelin also stimulates $\mathrm{GH}$ secretion in normal weight children and adults under fasting conditions. ${ }^{10}$ The effects of ghrelin on $\mathrm{GH}$ secretion in obese patients are less clear. ${ }^{10}$ Ghrelin-depen- 
dent $\mathrm{GH}$ secretion promotes hepatic gluconeogenesis and plays a central role in the defence against hypoglycaemia in states of nutrient deprivation. ${ }^{11,12}$ The aetiology of hyperghrelinaemia in PWS remains unclear, but at least some affected children are hyperghrelinaemic prior to the accretion of fat mass. ${ }^{4,13-15}$

Fasting insulin levels and HOMA-IR are lower and adiponectin levels higher in children with PWS than in age- and gendermatched obese controls. ${ }^{4,16}$ HOMA-IR ${ }^{17}$ is a measure of hepatic insulin sensitivity equal to the product of fasting blood glucose $(\mathrm{mg} / \mathrm{dl})$ and insulin $(\mu \mathrm{U} / \mathrm{ml})$ divided by 405 . While the hypoinsulinaemia, decreased HOMA-IR and hyperadiponectinaemia in PWS suggest heightened insulin sensitivity, the relative hyperghrelinaemia in PWS may contribute to hyperphagia. Nevertheless, the regulation of insulin, adiponectin and ghrelin in PWS is poorly understood.

To identify metabolic determinants of adiponectin and ghrelin in PWS, we conducted a systematic study of plasma amino acids, fatty acids, and amino acid and fatty acid metabolites in GHtreated and untreated PWS children and BMI-matched obese and lean controls. We focused on amino acid and fatty acid metabolites because previous studies showed roles for BCAA in the regulation of insulin sensitivity and food intake in obese adults and adolescents. ${ }^{18,19}$ We hypothesized that metabolic profiles in patients with PWS might account for their relative hyperghrelinaemia, hyperphagia and increased insulin sensitivity.

\section{Methods and procedures}

\section{Subjects}

Fourteen children with genetically confirmed PWS and normal glucose tolerance were enrolled. Healthy controls were recruited from local community paediatricians and the obesity- and insulin-resistant clinics at Duke University Medical Center. Controls included 14 healthy children with BMI $>95$ th percentile for age and gender (obese controls, OC) and 14 children of normal weight (lean controls, LC) of comparable age and gender. Nine PWS subjects ( 5 males and 4 females) were taking GH (mean $0.02 \mathrm{mg} / \mathrm{kg} /$ day) during the study.

The study was approved by the Duke IRB. A parent of each child gave written informed consent; when appropriate, each child provided assent before enrolment.

\section{Blood sample collection and processing}

Blood samples were collected between 7:30 and 10:00 am after an observed 12-h overnight fast. Samples in EDTA-treated and serum-separator tubes were immediately treated with Aprotinin, $500 \mathrm{KIU} / \mathrm{ml}$ [Roche, Indianapolis, IN, USA]. Serum and plasma were separated by centrifugation ( $1500 \mathrm{~g})$; all samples were aliquoted and stored at $-70{ }^{\circ} \mathrm{C}$ until assayed.

\section{Plasma acylcarnitines and amino acids}

Proteins were removed by precipitation with methanol. The aliquoted supernatants were dried and esterified with hot, acidic methanol (acylcarnitines) or n-butanol (amino acids). Acylcarnitines and amino acids were analysed by tandem mass spectrometry using stable isotope-labelled internal standards as previously described. $^{11}$

\section{Free fatty acids}

Individual free fatty acids were methylated with iodomethane, partially purified via solid-phase extraction and measured by gas chromatography/mass spectrometry. ${ }^{18,20}$

\section{Conventional metabolite analysis}

Plasma glucose and total free fatty acids (Roche Diagnostics, Indianapolis, IN, USA) were measured on a Hitachi 911 clinical analyzer.

\section{Measurement of ghrelin, insulin, adiponectin, leptin and PYY}

Plasma insulin concentrations were measured in all groups with a two-site IRMA method. ${ }^{13}$ The intra-assay and interassay $\mathrm{CV}$ were $3-5 \%$ and $5-7 \%$. The normal range was $0-69.5 \mathrm{pmol} / \mathrm{l}$. Total and HMW adiponectin levels were measured by enzyme immunoassay (Alpco Diagnostics, Salem, NH, USA). The intraassay CV was 5\% for total and HMW adiponectin. The interassay CV was $5 \%$ for total adiponectin and 6\% for HMW adiponectin. The sensitivity was $0.075 \mathrm{ng} / \mathrm{ml}$.

Plasma ghrelin was measured with a commercial RIA (Phoenix Pharmaceuticals, Belmont, CA, USA). The intra-assay and interassay coefficients of variation (CV) were $7 \%$ and $10 \%$, respectively. The lower and upper limits of detection for this assay were 24 and $1516 \mathrm{pmol} / \mathrm{l}$. Plasma leptin levels were determined using an RIA from Linco (St. Charles, MO, USA) with detection limit $0.5 \mathrm{ng} / \mathrm{ml}$ and intra-assay and interassay CV 2 and 5\%, respectively. Plasma total PYY was measured by RIA (Linco); intra-assay and interassay CV were $3-9 \%$ and $5-8 \%$, respectively.

\section{Statistical analysis}

The outcome variables among the PWS, OC and LC groups were compared using analysis of variance, nonparametric Mann-Whitney and Kruskal-Wallis tests. Correlations between ghrelin, adiponectin and BMI-z, HOMA-IR, plasma amino acids, and fatty acid metabolites were determined by Spearman rank order correlation. All statistical analyses were performed using GraphPad Prism software (GraphPad Software, Inc., La Jolla, CA, USA) and SigmaPlot software (Systat Software, Inc., Chicago, IL, USA).

\section{Results}

Characteristics of the study participants are shown in Table 1. The mean age of the PWS girls was less than that of the boys and girls in the other groups, but the difference was not statisti- 
Table 1. Auxologic and metabolic parameters of the three study cohorts (PWS, OC and OC) and comparison of GH-treated (PWS + GH) and untreated PWS subjects (PWS no GH)

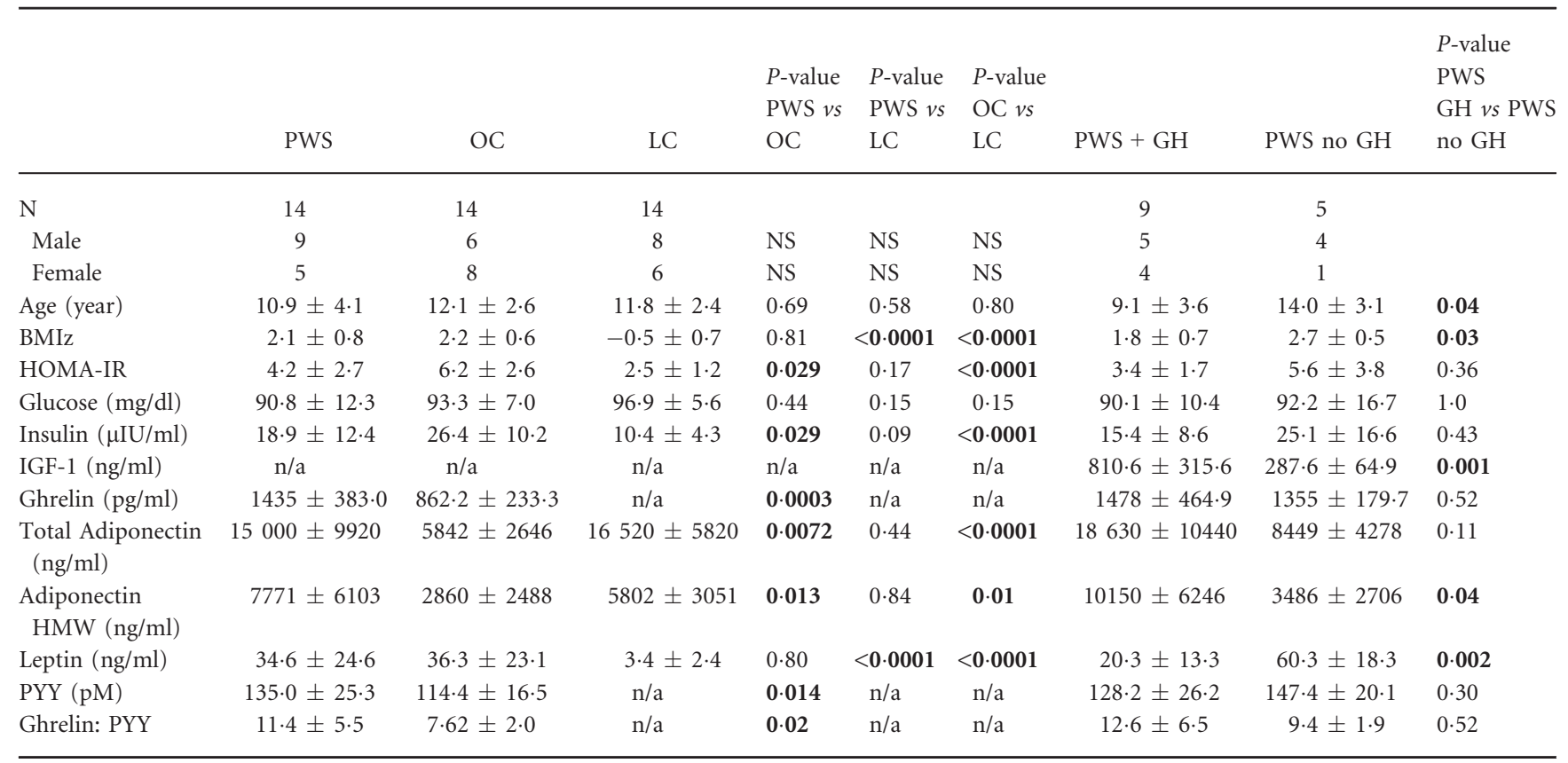

Serum ghrelin was not measured in LC. Data are means \pm SD unless otherwise noted. n/a indicates data not available. NS indicates not statistically significant. Values in bold indicated $P<0 \cdot 05$.

cally significant. BMI z-scores were comparable between PWS and OC groups.

\section{Baseline fasting insulin, ghrelin, adiponectin and PYY}

Despite comparable BMI z-scores, the cohort of PWS subjects had lower fasting insulin levels and HOMA-IR and higher fasting ghrelin, adiponectin (total and HMW) and peptide YY (PYY) than OC (Table 1). The ratio of ghrelin/PYY was higher in PWS than in OC. The fasting insulin, HOMA-IR, and total and high molecular weight (HMW) adiponectin levels in PWS were comparable to those in LC (Table 1), while leptin levels were markedly higher. Ghrelin was not measured in the LC cohort in this study, but has previously been reported in another group of lean children. ${ }^{13}$

\section{Effects of gender on baseline characteristics}

Important sex-dependent anthropometric and metabolic differences may be obscured when data from males and females are analysed collectively. ${ }^{19}$ Table 2 shows auxologic and hormonal data analysed separately in males and females. Relative hyperghrelinaemia was noted in both PWS males $(P=0.049)$ and females $(P=0.002)$, but was more striking in PWS females

Table 2. Auxologic and metabolic parameters segregated by gender. Serum ghrelin was not measured in LC

\begin{tabular}{|c|c|c|c|c|c|c|}
\hline & PWS F $(n=5)$ & PWS M $(n=9)$ & OC F $(n=8)$ & $\mathrm{OC} \mathrm{M}(n=6)$ & LC F $(n=6)$ & $\mathrm{LC} \mathrm{M}(n=8)$ \\
\hline Age (year) & $8 \cdot 8 \pm 4 \cdot 8$ & $12 \cdot 0 \pm 3 \cdot 5$ & $12 \cdot 9 \pm 3 \cdot 0$ & $11 \cdot 0 \pm 1.9$ & $10 \cdot 8 \pm 1 \cdot 9$ & $12 \cdot 5 \pm 2 \cdot 7$ \\
\hline $\mathrm{BMIz}$ & $1.9 \pm 0.69$ & $2 \cdot 3 \pm 0.8 \dagger \dagger$ & $2 \cdot 4 \pm 0 \cdot 2$ & $1 \cdot 8 \pm 0 \cdot 7$ & $-0.2 \pm 0.7$ & $-0.7 \pm 0.7$ \\
\hline HOMA-IR & $2 \cdot 8 \pm 0 \cdot 9 \S$ & $4 \cdot 9 \pm 3 \cdot 1$ & $6 \cdot 9 \pm 2 \cdot 5$ & $5 \cdot 1 \pm 2 \cdot 7$ & $2 \cdot 3 \pm 0.7$ & $2 \cdot 7 \pm 1 \cdot 3$ \\
\hline Glucose (mg/dl) & $93 \cdot 7 \pm 15 \cdot 2$ & $89 \cdot 3 \pm 11 \cdot 2$ & $94 \cdot 2 \pm 8 \cdot 9$ & $92 \cdot 1 \pm 3 \cdot 8$ & $95 \cdot 0 \pm 4 \cdot 5$ & $98 \cdot 3 \pm 6 \cdot 2$ \\
\hline Insulin $(\mu \mathrm{U} / \mathrm{ml})$ & $11 \cdot 9 \pm 3 \cdot 7 \S$ & $22 \cdot 8 \pm 14 \cdot 1$ & $29 \cdot 4 \pm 9 \cdot 0$ & $22 \cdot 4 \pm 11 \cdot 1$ & $9 \cdot 8 \pm 2 \cdot 7$ & $10 \cdot 8 \pm 5 \cdot 4$ \\
\hline Ghrelin $(\mathrm{pg} / \mathrm{ml})$ & $1529 \pm 467 \cdot 7 \S$ & $1382 \pm 346 \cdot 8^{\star *}$ & $753 \cdot 0 \pm 137 \cdot 7$ & $1008 \pm 265 \cdot 2$ & $\mathrm{n} / \mathrm{a}$ & $\mathrm{n} / \mathrm{a}$ \\
\hline Adiponectin (ng/ml) & $16070 \pm 5575 \S$ & $14400 \pm 11970$ & $4442 \pm 1491$ & $7709 \pm 2787$ & $17120 \pm 7267$ & $16070 \pm 4964$ \\
\hline Adiponectin HMW (ng/ml) & $8207 \pm 4164 \S$ & $7529 \pm 7189$ & $1506 \pm 1138 \dagger$ & $4665 \pm 2726$ & $7002 \pm 3637$ & $4902 \pm 2384$ \\
\hline Leptin $(\mathrm{ng} / \mathrm{ml})$ & $34 \cdot 4 \pm 28 \cdot 3$ & $34 \cdot 8 \pm 24 \cdot 2 \dagger \dagger$ & $49 \cdot 4 \pm 19 \cdot 4 \dagger$ & $18 \cdot 8 \pm 14 \cdot 7$ & $5 \cdot 3 \pm 2 \cdot 8 t$ & $2 \cdot 0 \pm 0 \cdot 4$ \\
\hline PYY (pM) & $131 \cdot 7 \pm 25 \cdot 3$ & $136 \cdot 8 \pm 27 \cdot 0$ & $110 \cdot 4 \pm 18 \cdot 8$ & $119 \cdot 8 \pm 12 \cdot 3$ & $\mathrm{n} / \mathrm{a}$ & $\mathrm{n} / \mathrm{a}$ \\
\hline Ghrelin: PYY & $12 \cdot 5 \pm 6 \cdot 5$ & $10 \cdot 8 \pm 5 \cdot 1$ & $7 \cdot 0 \pm 1.9$ & $8.4 \pm 1.9$ & $\mathrm{n} / \mathrm{a}$ & $\mathrm{n} / \mathrm{a}$ \\
\hline
\end{tabular}

Data are means $\pm \mathrm{SD}$ unless otherwise noted. $\mathrm{n} / \mathrm{a}$ indicates data not available. $P<0.05$ for $\dagger$ OC females $v s$ OC males; $\ddagger$ LC females $v s$ LC males; $\S$ PWS females vs OC females; $₫$ PWS females $v s$ LC females; ${ }^{*}$ PWS males $v s$ OC males; $\dagger \dagger$ PWS males $v s$ LC males. 
than PWS males. Ghrelin levels were twofold higher in PWS females than OC females, but only 37\% higher in PWS males than OC males. There were no gender differences in the levels of PYY among PWS males and females or OC males and females. Fasting insulin levels and HOMA-IR were lower $(P=0.002)$ and total and HMW adiponectin levels higher $(P=0.002)$ in PWS females than OC females and were comparable to those in LC females. In contrast, fasting insulin and HOMA-IR in PWS males were comparable to those in OC males. Total and HMW adiponectin levels in PWS males were highly variable and did not differ significantly from those in OC or LC males. Leptin levels in female LC and OC were higher than those in LC and OC males. In contrast, leptin levels were similar in PWS females and males. Consequently, leptin levels were significantly higher in PWS males than LC males.

\section{Plasma amino acids in PWS, OC, LC}

Plasma amino acids were analysed by group (PWS, OC and LC) and gender. The levels of glycine, histine, serine, Asx (aspartate/asparagine), citrulline and arginine were lower in OC than in LC, while the levels of tyrosine (Tyr) and Glx (glutamate/glutamine) were higher (Table 3). Tyr levels in PWS were lower than those in OC and comparable to those in LC; Glx levels in PWS were also lower than those in OC, but higher than those in LC.

To determine whether amino acid levels in the various cohorts vary according to gender, we analysed plasma amino acids separately in males and females. Table 4 demonstrates significant and differential gender effects on the levels of the BCAA Leu/Ile and Val in the three cohorts. The levels of Leu/Ile were higher in LC males than in LC females. However, levels of Leu/Ile were higher in OC females than in LC females and were comparable to those in OC males. Interest- ingly, the levels in Leu/Ile in PWS females were comparable to those in LC females and lower than those in OC females. Likewise, Val levels in PWS females were lower than those in PWS males.

In contrast to Leu/Ile, the levels of Glx were lower in both PWS males and females than in OC males and females. Glx levels in PWS were comparable to those in LC males, while Glx levels in PWS females were higher than those in LC females.

Tyr levels in the PWS cohort were lower than those in OC and LC (Table 3). This reflects the low levels of Tyr in PWS females, but not males (Table 4). Alanine, methionine, proline and arginine levels were also lower in PWS females than OC and LC females.

\section{Free fatty acids}

The levels of various free fatty acids in PWS were compared to those in OC and LC (Supplemental Table 1). Most striking were the high levels of C16:1 palmitoleic acid in PWS and of C18:3 alpha-linolenic acid in PWS and OC. Relative to LC, PWS also had higher levels of myristic, palmitic and linoleic acids, while OC had higher arachidonic acid levels. However, there were no significant differences between the PWS and OC groups in levels of any free fatty acids. Interestingly, unlike the levels of BCAA, the levels of fatty acids in PWS did not vary in either a gender-dependent (Table S2) or GH-dependent manner (Table S3).

\section{Acylcarnitines}

By-products of BCAA (and methionine) catabolism include propionyl-L-carnitine (C3) and valeryl-L-carnitine (C5); in contrast, acetylcarnitine (C2) is an end-product of fatty acid oxidation.

Table 3. Amino acid levels in the three study cohorts (PWS, OC and LC) and comparison of GH-treated (PWS + GH) and untreated PWS subjects (PWS no GH)

\begin{tabular}{|c|c|c|c|c|c|c|c|c|c|}
\hline $\begin{array}{l}\text { Amino } \\
\text { Acid }(\mu \mathrm{M})\end{array}$ & PWS & OC & LC & $\begin{array}{l}\text { P-value } \\
\text { PWS vs } \\
\text { OC }\end{array}$ & $\begin{array}{l}\text { P-value } \\
\text { PWS vs LC }\end{array}$ & $\begin{array}{l}\text { P-value } \\
\text { OC vs LC }\end{array}$ & PWS + GH & PWS no GH & $\begin{array}{l}\text { P-value PWS } \\
\mathrm{GH} \text { vs no } \mathrm{GH}\end{array}$ \\
\hline $\mathrm{N}$ & 14 & 14 & 14 & & & & 9 & 3 & \\
\hline Leu/ILE & $146 \cdot 4 \pm 41 \cdot 0$ & $161 \cdot 1 \pm 15 \cdot 1$ & $150 \cdot 1 \pm 26 \cdot 8$ & $0 \cdot 23$ & $0 \cdot 83$ & $0 \cdot 15$ & $137 \cdot 1 \pm 42 \cdot 3$ & $174 \cdot 3 \pm 22 \cdot 7$ & $0 \cdot 10$ \\
\hline Val & $209 \cdot 8 \pm 55 \cdot 1$ & $215 \cdot 4 \pm 26 \cdot 7$ & $203 \cdot 9 \pm 33 \cdot 3$ & $0 \cdot 87$ & 0.66 & $0 \cdot 56$ & $194.9 \pm 53.5$ & $254 \cdot 3 \pm 35 \cdot 3$ & $0 \cdot 14$ \\
\hline Phe & $64 \cdot 7 \pm 20 \cdot 6$ & $64 \cdot 8 \pm 6 \cdot 2$ & $59.2 \pm 9.8$ & 0.69 & $0 \cdot 41$ & $0 \cdot 056$ & $60 \cdot 7 \pm 21 \cdot 3$ & $77 \cdot 0 \pm 14 \cdot 9$ & $0 \cdot 10$ \\
\hline Tyr & $67 \cdot 9 \pm 21 \cdot 7$ & $82 \cdot 4 \pm 10 \cdot 9$ & $71 \cdot 1 \pm 9 \cdot 3$ & 0.03 & $0 \cdot 48$ & $0 \cdot 0169$ & $58 \cdot 0 \pm 12 \cdot 8$ & $97 \cdot 7 \pm 13 \cdot 0$ & $0 \cdot 009$ \\
\hline Gly & $344 \cdot 7 \pm 164 \cdot 3$ & $284 \cdot 9 \pm 51 \cdot 2$ & $420 \cdot 5 \pm 63 \cdot 8$ & $0 \cdot 12$ & $0 \cdot 0008$ & $<0.0001$ & $356 \cdot 4 \pm 189 \cdot 3$ & $309 \cdot 3 \pm 50 \cdot 7$ & $0 \cdot 72$ \\
\hline Ala & $313 \cdot 9 \pm 105 \cdot 2$ & $393 \cdot 5 \pm 100 \cdot 2$ & $383 \cdot 0 \pm 92 \cdot 7$ & $0 \cdot 13$ & $0 \cdot 14$ & $0 \cdot 81$ & $287 \cdot 1 \pm 98 \cdot 8$ & $394 \cdot 3 \pm 94 \cdot 1$ & $0 \cdot 21$ \\
\hline Met & $28 \cdot 1 \pm 7 \cdot 7$ & $30 \cdot 4 \pm 3 \cdot 4$ & $31 \cdot 1 \pm 4 \cdot 9$ & $0 \cdot 17$ & $0 \cdot 18$ & 0.69 & $25 \cdot 0 \pm 5 \cdot 4$ & $37 \cdot 3 \pm 5 \cdot 8$ & 0.009 \\
\hline His & $73 \cdot 5 \pm 12 \cdot 4$ & $81 \cdot 0 \pm 11 \cdot 1$ & $87 \cdot 4 \pm 5 \cdot 9$ & $0 \cdot 23$ & 0.0006 & 0.0387 & $73 \cdot 4 \pm 14 \cdot 2$ & $73 \cdot 7 \pm 6 \cdot 1$ & $0 \cdot 72$ \\
\hline Ser & $102 \cdot 3 \pm 18 \cdot 3$ & $106 \cdot 8 \pm 14 \cdot 9$ & $122 \cdot 1 \pm 8 \cdot 5$ & $0 \cdot 97$ & 0.0013 & 0.0024 & $99 \cdot 8 \pm 19.7$ & $109 \cdot 7 \pm 13 \cdot 5$ & $0 \cdot 48$ \\
\hline Pro & $157 \cdot 8 \pm 44 \cdot 7$ & $171 \cdot 2 \pm 44 \cdot 2$ & $201 \cdot 1 \pm 45 \cdot 4$ & 0.48 & 0.02 & $0 \cdot 16$ & $149 \cdot 1 \pm 45 \cdot 7$ & $183 \cdot 7 \pm 35 \cdot 8$ & $0 \cdot 21$ \\
\hline Asx & $88 \cdot 7 \pm 26 \cdot 7$ & $80 \cdot 1 \pm 19 \cdot 5$ & $107 \cdot 3 \pm 33 \cdot 8$ & $0 \cdot 45$ & $0 \cdot 08$ & 0.0308 & $82 \cdot 1 \pm 27 \cdot 6$ & $108 \cdot 3 \pm 9 \cdot 5$ & $0 \cdot 1$ \\
\hline Glx & $130 \cdot 7 \pm 43 \cdot 1$ & $172 \cdot 6 \pm 43 \cdot 5$ & $85 \cdot 5 \pm 38 \cdot 5$ & 0.01 & $0 \cdot 0026$ & $<0.0001$ & $138 \cdot 3 \pm 38 \cdot 2$ & $107 \cdot 7 \pm 57 \cdot 8$ & $0 \cdot 28$ \\
\hline Orn & $66 \cdot 2 \pm 24 \cdot 3$ & $49 \cdot 3 \pm 6 \cdot 0$ & $50 \cdot 8 \pm 11 \cdot 6$ & $0 \cdot 15$ & $0 \cdot 11$ & $0 \cdot 908$ & $62 \cdot 6 \pm 24 \cdot 5$ & $77 \cdot 0 \pm 24 \cdot 6$ & $0 \cdot 37$ \\
\hline Cit & $26 \cdot 4 \pm 7 \cdot 1$ & $25 \cdot 6 \pm 6 \cdot 2$ & $34 \cdot 3 \pm 3 \cdot 7$ & $0 \cdot 72$ & 0.0034 & 0.0007 & $26 \cdot 2 \pm 7 \cdot 8$ & $27 \cdot 0 \pm 5 \cdot 6$ & $1 \cdot 0$ \\
\hline Arg & $76 \cdot 6 \pm 32 \cdot 8$ & $87 \cdot 4 \pm 8 \cdot 2$ & $109 \cdot 2 \pm 18 \cdot 0$ & $0 \cdot 62$ & 0.01 & 0.0023 & $72 \cdot 6 \pm 37 \cdot 4$ & $88 \cdot 7 \pm 3 \cdot 5$ & $0 \cdot 86$ \\
\hline
\end{tabular}

Values are means \pm SD. Values in bold indicated $P<0.05$. 
Table 4. Amino acid levels analysed by gender

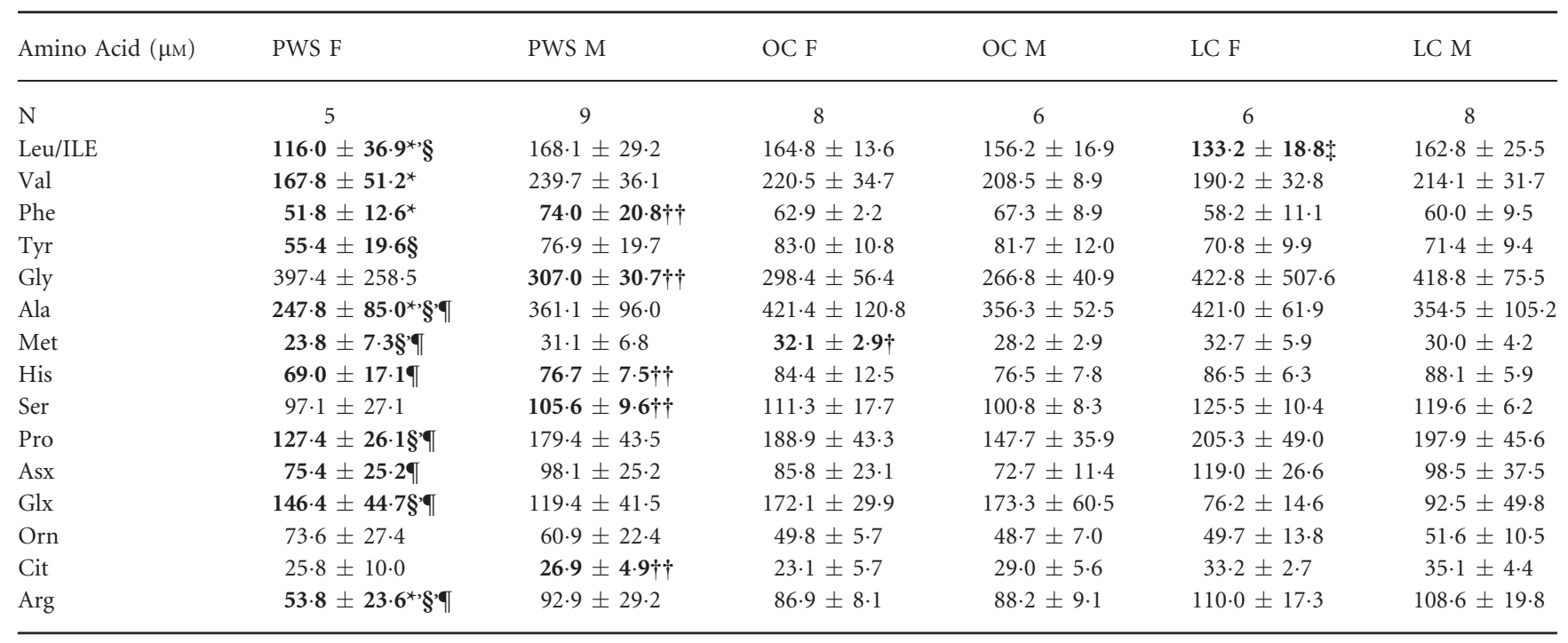

Values are means \pm SD. $P<0.05$ for ${ }^{*}$ PWS females $v s$ PWS males; $\dagger$ OC females $v s$ OC males; $\$$ LC females $v s$ LC males; $\S$ PWS females $v s$ OC females; qPWS females $v s$ LC females; $\dagger \dagger$ PWS males $v$ L LC males. Values in bold indicated $P<0.05$.

Relative to LC, OC had lower levels of C2 (acetyl) carnitine (Table S4). In contrast, C2 levels in PWS were comparable to those in LC. C2 levels in PWS appeared to be independent of gender or GH therapy (Tables S5 and S6).

There were no significant differences in C3 or C5 acylcarnitines among the groups, but the ratio of the sum of $\mathrm{C} 3+\mathrm{C} 5$ to the sum of acylcarnitines was higher in PWS and OC than LC. This ratio was selectively higher in PWS males and OC males and was not influenced by GH treatment (Tables S5 and S6). Collectively, these findings may suggest lower rates of complete fatty acid oxidation in OC, preservation of complete fatty oxidation in PWS, and increased rates of BCAA and methionine catabolism in PWS and OC relative to LC.

\section{Effect of GH treatment on auxologic parameters, hormones and adipocytokines}

Nine of 14 PWS subjects were treated with GH. A comparison of GH-treated and untreated PWS subjects is shown in Table 1. PWS subjects treated with GH (5 males and 4 females) were younger in age (mean 9.11 vs 14.0 years) than untreated PWS subjects (4 males and 1 female) and had lower BMI-z. However, fasting glucose, insulin, HOMA-IR, ghrelin and PYY were comparable. These findings suggest that the relative hypoinsulinaemia, hyperghrelinaemia, elevated PYY and increased ghrelin/ PYY do not result from GH treatment. There was considerable variability in the total adiponectin levels in both the GH-treated and untreated patients. While the GH-treated had higher mean adiponectin levels, the difference was not statistically significant. On the other hand, PWS subjects treated with GH had increased levels of HMW adiponectin $(P=0 \cdot 04)$. IGF-1 levels were higher $(P=0.001)$, and leptin levels were lower in GH-treated subjects $(P=0 \cdot 002)$.

\section{Effects of $\mathrm{GH}$ on plasma amino acids}

We considered the possibility that differences in amino acid levels in PWS might be related to GH therapy. PWS children treated with GH (5 males and 4 females) had lower levels of Tyr $(P=0.009)$ and Met $(P=0.009)$ than untreated PWS (2 males and 1 female) (Table 3$)$. Table 5 demonstrates that the levels of BCAA $(P=0.016)$ and Tyr $(P=0.032)$ were lower in GH-treated PWS females $(n=4)$ than in GH-treated PWS males $(n=5)$. These findings suggest that GH may exert differential effects on amino acids in male and female patients with PWS; alternatively, the sex differences in amino acids may be independent of GH therapy.

\section{Auxologic and metabolic correlates of ghrelin and adiponectin}

In PWS subjects, ghrelin correlated inversely with BMI-z $(r=-0.59, P=0.027)$, HOMA-IR $(r=-0.55, P=0.042)$, and the levels of Leu/Ile and Val $(r=-0.629, P=0.028$ and $r=-0.629, P=0.028$, respectively). In OCs, ghrelin correlated inversely with BMIz $(r=-0.59, P=0.029)$ and HOMA-IR $(-0.53, P=0.049)$, but not with Leu/Ile or Val $(r=-0 \cdot 32$, $P=0.266$ and $r=0.12, \quad P=0.673$, respectively, Table S7, Fig. 1). Adiponectin correlated negatively with Leu/Ile and Val in OC $(r=-0.62, P=0.017) ; r=-0.50, P=0.06$, respectively); in contrast, adiponectin correlated inversely with $\mathrm{BMIz}$ and HOMA-IR in PWS $(r=-0.61, P=0.019$ and $r=-0.78$, $P=0.0009$, respectively, Table S7, Fig. 1). The inverse correlation between adiponectin and HOMA-IR in PWS was strengthened by natural $\log$ transformation of the data (Fig. 1f). The most powerful determinants of ghrelin in PWS were the levels of BCAA, while the most powerful determinants of ghrelin in 
Table 5. Comparison of metabolic profiles in GH-treated PWS males (M) and females (F)

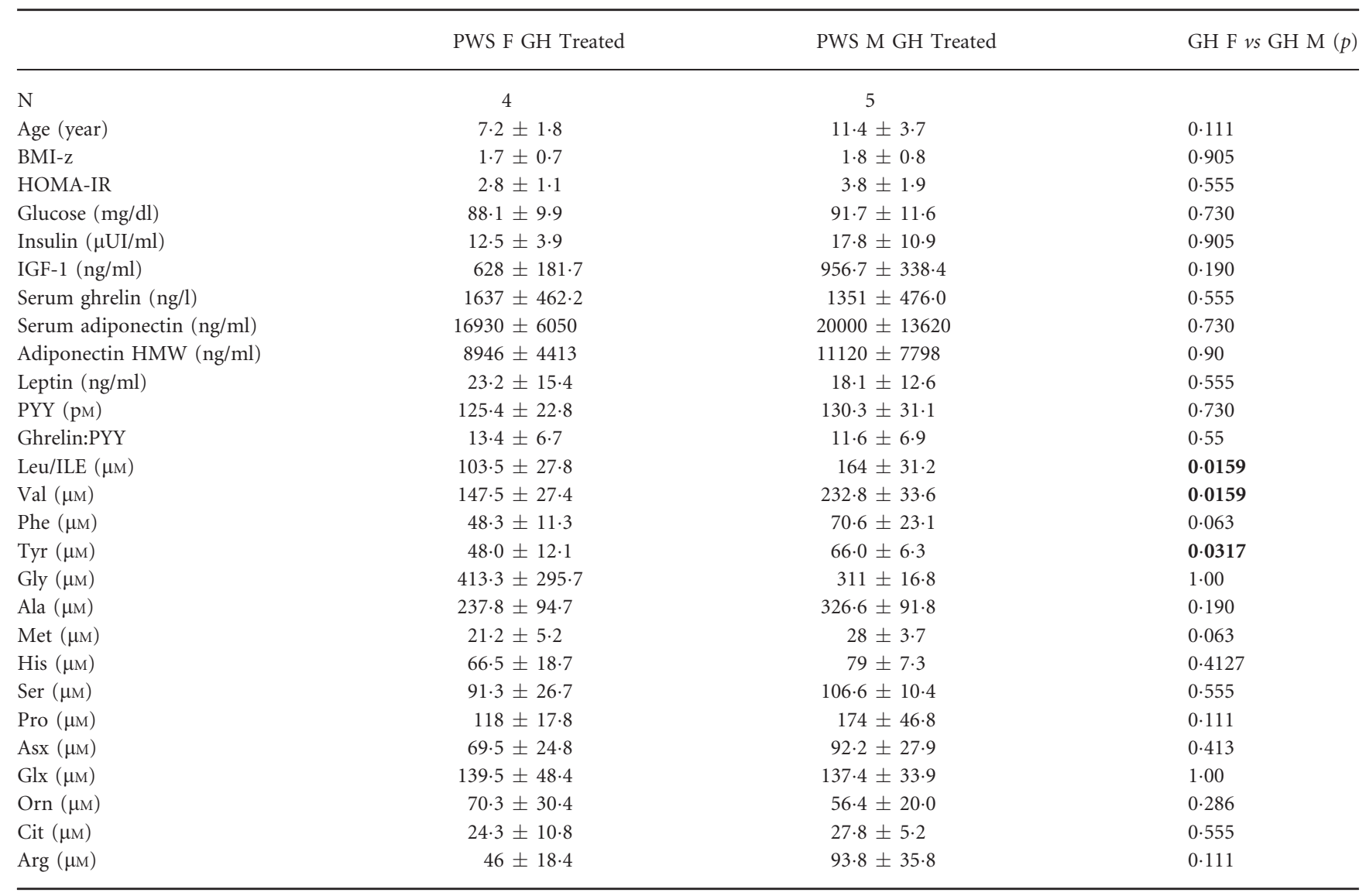

Values are means $\pm \mathrm{SD}$. Values in bold indicated $P<0.05$.

OC were BMIz and HOMA-IR. Conversely, the strongest determinants of adiponectin in PWS were BMIz and HOMA-IR, but the strongest determinants of adiponectin in OC were the levels of Leu/Ile and Val (Table S7).

\section{Discussion}

'Exogenous' obesity is associated with hypoghrelinaemia, hypoadiponectinaemia, insulin resistance and compensatory hyperinsulinaemia. In contrast, PWS combines hyperghrelinaemia, hyperphagia and obesity with relative hyperadiponectinaemia and hypoinsulinaemia. The factors accounting for the metabolic differences between patients with PWS and exogenous obesity are unclear. Here, we report striking differences in amino acids, fatty acids, and amino acid and fatty acid metabolites among male and female PWS and OC children and differential regulation of ghrelin and adiponectin by BMIz, HOMA-IR and the BCAA. We speculate that these metabolic differences may reflect, or contribute to, the relative hyperghrelinaemia, hyperadiponectinaemia and hypoinsulinaemia of PWS and may therefore play roles in the development and/or progression of hyperphagia and weight gain and the maintenance of insulin sensitivity.

Numerous studies from our group and others find that fasting ghrelin levels in PWS are higher than those in age- and BMImatched OC. ${ }^{4,13,14,21}$ In contrast, the relative levels of PYY in
PWS are more variable. ${ }^{16,21,22}$ We found that fasting PYY levels are mildly elevated in PWS; however, the fasting ratio of ghrelin/ PYY, which may be considered a marker of orexigenic drive, is also high. This is primarily driven by the higher levels of ghrelin in PWS. Interestingly, while ghrelin and PYY levels were comparable in PWS males and females, the relative hypoinsulinaemia and hyperadiponectinaemia of PWS are more characteristic of females than males. Likewise, Leu/Ile, Val, Tyr, Met, Ala, Glx and Arg levels are lower in PWS females than either PWS males or OC females and (with the exception of Glx) are comparable to those in LC females. In contrast, the levels of BCAA and various other amino acids in PWS males are comparable to those in OC males; the major exception is Glx, which is lower in PWS males than in OC males.

In a previous investigation, ${ }^{19}$ we found that BCAA levels in healthy obese adolescent males were higher than in obese adolescent females. The teenagers were older in that study (mean age 14 year) than in the current study and their BCAA levels were significantly higher. In the current study, BCAA levels are higher in LC males than LC females. Our study shows that the sex differences in BCAA levels observed in LC are abolished in 'exogenous' obesity, but preserved in children with PWS. Interestingly, the levels of fatty acids and acylcarnitines do not differ significantly between PWS boys and girls. Factors that may contribute to these variable sex-dependent metabolic differences among PWS, 
(a) PWS ghrelin vs BMIz

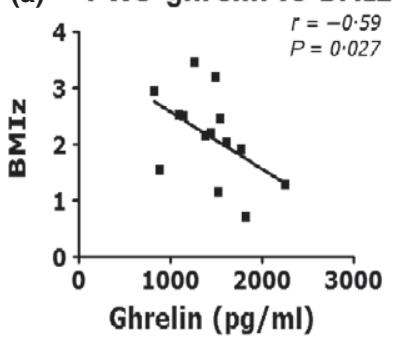

(c) PWS ghrelin vs Leu/Ile

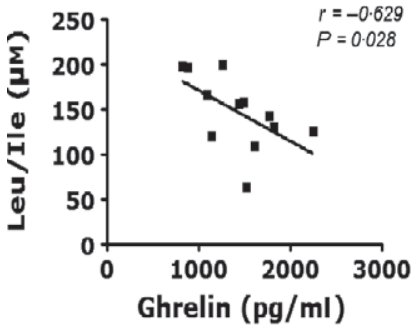

(e) PWS adiponectin vs BMIz

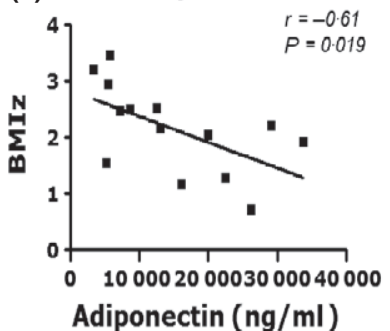

(g) PWS adiponectin vs Leu/Ile

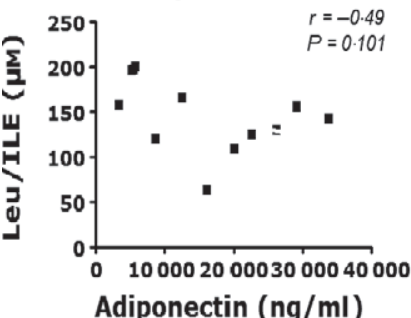

(b) PWS ghrelin vs HOMA-IR

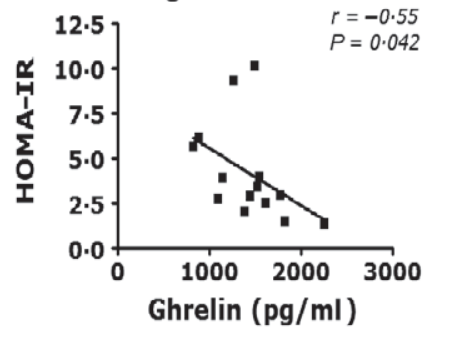

(d) PWS ghrelin vs Val

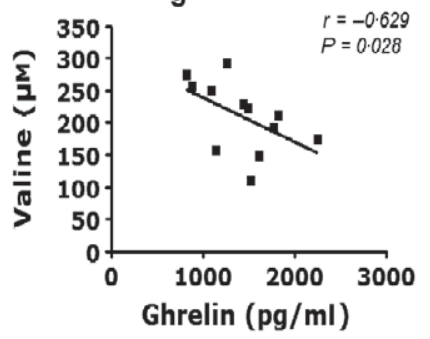

(f) PWS adiponectin vs HOMA IR

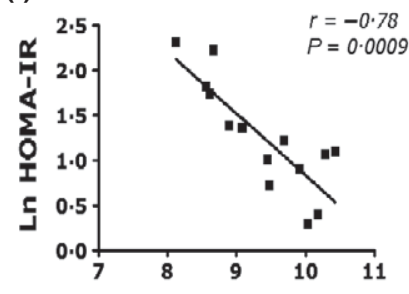

Ln Adiponectin (ng/ml)

(h) PWS adiponectin vs Val

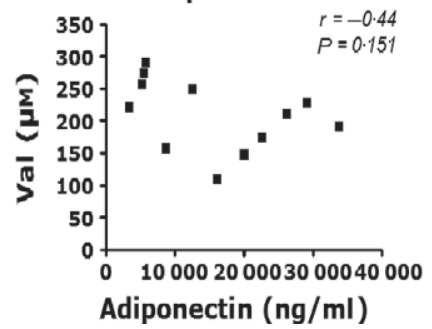

Fig. 1 Correlation of ghrelin and adiponectin with auxologic and metabolic parameters in PWS subjects. (a-d): relationship of fasted ghrelin in PWS subjects to: (a) BMI-z; (b) HOMA-IR; and (c, d) branch-chain amino acids. (e-h): relationship of fasted adiponectin levels in PWS subjects to: (e) BMI-z; (f) HOMA-IR; and (g, h) branch-chain amino acids. The data in if are natural logtransformed.
OC and LC children are unclear but could include variations in sex steroid and GH production, fat distribution, lean body mass and/or autonomic function. ${ }^{19,23-25}$ In particular, the effects of differential fat distribution may be a determining factor in the unique metabolic profile of PWS. Prior studies have shown that visceral fat stores are lower in PWS females than in OC females; the reduction in visceral fat is associated with lower levels of insulin and HOMA-IR. In contrast, subcutaneous fat stores in PWS females are comparable to those in OC females. ${ }^{25}$ It should be noted the PWS females were younger than PWS males. Previous studies showed that HOMA-IR levels in normal weight prepubertal children are lower than those in older children ${ }^{26}$; therefore, age or pubertal development may have impacted our results.

In theory, the metabolic differences between PWS and OC may reflect, or may contribute to, the development or progression of, certain clinical features of PWS. For example, the major determinants of fasting ghrelin in PWS were the levels of BCAAs, while the most powerful determinants of ghrelin in OC were BMIz and HOMA-IR. Conversely, the strongest determinants of adiponectin in PWS were BMIz and HOMA-IR, but the strongest determinants of adiponectin in OC were the levels of Leu/Ile and Val. As leucine administration decreases food intake in rodents and humans, ${ }^{27,28}$ we speculate that low levels of BCAA in PWS females may promote or facilitate their hyperghrelinaemia and hyperphagia. Low levels of leucine might also reduce mTOR signalling and thereby increase insulin sensitivity, particularly in PWS females. ${ }^{29}$ The relative insulin hypersensitivity of PWS may also be maintained by high levels of adiponectin, which increases hepatic insulin sensitivity and fatty acid oxidation. ${ }^{16,24,25}$ This may explain why C2 (acetyl) carnitine levels in PWS are comparable to those in LC and higher than those in OC. An increase in the ratio of [C3 $+\mathrm{C} 5]$ to sum of acylcarnitines might be a marker for proteolysis and/or amino acid catabolism in PWS males, predisposing to sarcopenia. 
A previous investigation showed lower levels of glutamine and higher levels of glutamic acid in PWS relative to obese controls, but no differences in the levels of BCAA or Tyr. ${ }^{30}$ However, that study did not analyse the role of gender and was conducted in young adults prior to the advent of growth hormone therapy. While the effects of GH on growth and body composition in PWS are well-established, ${ }^{31,32}$ the effects of the hormone on metabolic function have not been studied extensively. Here, we show that the levels of hormones and metabolites in PWS children are modulated by GH therapy. We found that GH treatment was associated with lower BMI Z-score, lower leptin, higher IGF-1 and higher levels of HMW adiponectin $(P=0 \cdot 04)$. These findings are consistent with studies showing that $\mathrm{GH}$ increases linear growth and reduces body fat stores in PWS. ${ }^{33,34}$ A reduction in body fat stores, reflected in the fall in leptin levels, may explain in part the increases in HMW adiponectin observed in GH-treated subjects. ${ }^{16,35}$ On the other hand, GH treatment of PWS patients had no effect on fasting insulin, ghrelin, PYY, free fatty acids or acylcarnitines.

The levels of Leu/Ile, Val and Tyr were lower in GH-treated PWS females than in GH-treated PWS males. Interestingly, a previous investigation showed that acute administration of $\mathrm{GH}$ to healthy young men reduced plasma Tyr, Ile and Val levels during a 7 -h infusion period. ${ }^{36} \mathrm{GH}$ therapy may selectively reduce the levels of BCAA and Tyr in PWS girls; alternatively, low BCAA and Tyr levels may be characteristic of PWS females and independent of GH treatment.

Limitations of our study include multiple comparisons of crosssectional data collected at a single point in time and analysis of total ghrelin rather than active and desacyl ghrelin. Our study included a small number of subjects treated with $\mathrm{GH}$, possibly limiting our ability to assess adequately the effects of GH on metabolic function. Moreover, the roles of dietary intake and macronutrient preference on metabolic function were not explored. Despite these limitations, our study compared a relatively large cohort (for a rare genetic disorder) of children with PWS with comparable groups of OC and LC.

Despite the relative insulin sensitivity of PWS subjects, their morbid obesity places them at risk of metabolic sequelae including type 2 diabetes. ${ }^{25}$ Future work on adiponectin analogues or other therapies aimed at upregulation of adiponectin receptors may prove clinically useful in limiting the metabolic sequelae of obesity, including insulin resistance, type 2 diabetes and metabolic syndrome.

\section{Acknowledgements}

We thank Juanita Cuffee and Christopher Newgard. Supported by grants from NIH T32 HD 43029-11A1 (KI), David and Cynthia King Pediatric Endocrine Fellowship Fund (KI, MF), Canadian Institutes of Health Research (AMH) and Foundation for Prader Willi Research (MF, AMH).

\section{Conflict of interest statement}

The authors have nothing to disclose.

\section{References}

1 Cassidy, S.B., Dykens, E. \& Williams, C.A. (2000) Prader-Willi and angelman syndromes: sister imprinted disorders. American Journal of Medical Genetics, 97, 137-146.

2 Butler, M.G. (2011) Prader-Willi syndrome: obesity due to genomic imprinting. Current Genomics, 12, 204-215.

3 Emerick, J. \& Vogt, K. (2013) Endocrine manifestations and management of Prader-Willi syndrome. International Journal of Pediatric Endocrinology, 2013, 14.

4 Haqq, A.M., Grambow, S.C., Muehlbauer, M. et al. (2008) Ghrelin concentrations in Prader-Willi syndrome (PWS) infants and children: changes during development. Clinical Endocrinology, 69, 911-920.

5 Prodam, F., Monzani, A., Ricotti, R. et al. (2014) Systematic review of ghrelin response to food intake in pediatric age, from neonates to adolescents. The Journal of Clinical Endocrinology \& Metabolism, 99, 1556-1568.

6 Wren, A.M., Seal, L.J., Cohen, M.A. et al. (2001) Ghrelin enhances appetite and increases food intake in humans. The Journal of Clinical Endocrinology \& Metabolism 86, 5992-5995.

7 Tschop, M., Smiley, D.L. \& Heiman, M.L. (2000) Ghrelin induces adiposity in rodents. Nature, 407, 908-913.

8 Batterham, R.L. \& Bloom, S.R. (2003) The gut hormone peptide YY regulates appetite. Annals of the New York Academy of Sciences, 994, 162-168.

9 Stock, S., Leichner, P., Wong, A.C.K. et al. (2005) Ghrelin, Peptide YY, glucose-dependent insulinotropic polypeptide, and hunger responses to a mixed meal in anorexic, obese, and control female adolescents. The Journal of Clinical Endocrinology \& Metabolism, 90, 2161-2168.

10 Nass, R., Gaylinn, B.D. \& Thorner, M.O. (2011) The role of ghrelin in GH secretion and GH disorders. Molecular and Cellular Endocrinology, 340, 10-14.

11 Bartz, S., Mody, A., Hornik, C. et al. (2014) Severe acute malnutrition in childhood: hormonal and metabolic status at presentation, response to treatment, and predictors of mortality. The Journal of Clinical Endocrinology \& Metabolism, 99, 21282137.

12 Li, R.L., Sherbet, D.P., Elsbernd, B.L. et al. (2012) Profound hypoglycemia in starved, ghrelin-deficient mice is caused by decreased gluconeogenesis and reversed by lactate or fatty acids. Journal of Biological Chemistry, 287, 17942-17950.

13 Haqq, A.M., Farooqi, I.S., O’Rahilly, S. et al. (2003) Serum ghrelin levels are inversely correlated with body mass index, age, and insulin concentrations in normal children and are markedly increased in Prader-Willi syndrome. The Journal of Clinical Endocrinology \& Metabolism, 88, 174-178.

14 Feigerlová, E., Diene, G., Conte-Auriol, F. et al. (2008) Hyperghrelinemia precedes obesity in Prader-Willi syndrome. The Journal of Clinical Endocrinology \& Metabolism, 93, 2800-2805.

15 Kweh, F.A., Miller, J.L., Sulsona, C.R. et al. (2014) Hyperghrelinemia in Prader-Willi syndrome begins in early infancy long before the onset of hyperphagia. American Journal of Medical Genetics Part A 167, 69-79.

16 Haqq, A.M., Muehlbauer, M., Svetkey, L.P. et al. (2007) Altered distribution of adiponectin isoforms in children with Prader-Willi syndrome (PWS): association with insulin sensitivity and circulating satiety peptide hormones. Clinical Endocrinology, 67, 944-951.

17 Muniyappa, R., Lee, S., Chen, H. et al. (2008) Current approaches for assessing insulin sensitivity and resistance in vivo: 
advantages, limitations, and appropriate usage. American Journal of Physiology - Endocrinology and Metabolism, 294, E15-E26.

18 Newgard, C.B., An, J., Bain, J.R. et al. (2009) A branched-chain amino acid-related metabolic signature that differentiates obese and lean humans and contributes to insulin resistance. Cell Metabolism, 9, 311-326.

19 Newbern, D., Gumus Balikcioglu, P., Balikcioglu, M. et al. (2014) Sex differences in biomarkers associated with insulin resistance in obese adolescents: metabolomic profiling and principal components analysis. The Journal of Clinical Endocrinology \& Metabolism, 99, 4730-4739.

20 Patterson, B.W., Zhao, G., Elias, N. et al. (1999) Validation of a new procedure to determine plasma fatty acid concentration and isotopic enrichment. Journal of Lipid Research, 40, 2118-2124.

21 Goldstone, A.P., Patterson, M., Kalingag, N. et al. (2005) Fasting and postprandial hyperghrelinemia in Prader-Willi syndrome is partially explained by hypoinsulinemia, and is not due to peptide YY3-36 deficiency or seen in hypothalamic obesity due to craniopharyngioma. The Journal of Clinical Endocrinology \& Metabolism, 90, 2681-2690.

22 Butler, M.G., Bittell, D.C. \& Talebizadeh, Z. (2004) Plasma peptide YY and ghrelin levels in infants and children with PraderWilli syndrome. Journal of Pediatric Endocrinology and Metabolism, 17, 1177-1184.

23 Haqq, A.M., Muehlbauer, M.J., Newgard, C.B. et al. (2011) The metabolic phenotype of Prader-Willi syndrome (PWS) in childhood: heightened insulin sensitivity relative to body mass index. The Journal of Clinical Endocrinology \& Metabolism, 96, E225-E232.

24 Haqq, A.M., DeLorey, D.S., Sharma, A.A. et al. (2012) Autonomic nervous system dysfunction in obesity and Prader-Willi syndrome: current evidence and implications for future obesity therapies. Clinical Obesity, 1, 175-183.

25 Goldstone, A.P., Thomas, E.L., Brynes, A.E. et al. (2001) Visceral adipose tissue and metabolic complications of obesity are reduced in Prader-Willi syndrome female adults: evidence for novel influences on body fat distribution. The Journal of Clinical Endocrinology \& Metabolism, 86, 4330-4338.

26 Peplies, J., Jimenez-Pavon, D., Savva, S.C. et al. (2014) Percentiles of fasting serum insulin, glucose, HbA1c and HOMA-IR in prepubertal normal weight European children from the IDEFICS cohort. International Journal of Obesity, 38(Suppl 2), S39-S47.
27 Laeger, T., Reed, S.D., Henagan, T.M. et al. (2014) Leucine acts in the brain to suppress food intake but does not function as a physiological signal of low dietary protein. American Journal of Physiology: Regulatory, Integrative and Comparative Physiology, 307, R310-R320.

28 Drgonova, J., Jacobsson, J.A., Han, J.C. et al. (2013) Involvement of the neutral amino acid transporter SLC6A15 and leucine in obesity-related phenotypes. PLoS ONE, 8, e68245.

29 Tai, E.S., Tan, M.L.S., Stevens, R.D. et al. (2010) Insulin resistance is associated with a metabolic profile of altered protein metabolism in Chinese and Asian-Indian men. Diabetologia, 53, 757-767.

30 Butler, M.G., Murrell, J.E. \& Greene, H.L. (1990) Amino acid levels in Prader-Willi syndrome and obese individuals. Dysmorphology and Clinical Genetics, 4, 18-22.

31 Deal, C.L., Tony, M., Höybye, C. et al. (2013) Growth hormone research society workshop summary: consensus guidelines for recombinant human growth hormone therapy in Prader-Willi syndrome. The Journal of Clinical Endocrinology \& Metabolism, 98, E1072-E1087.

32 Kuromaru, R., Kohno, H., Ueyama, N. et al. (1999) Long-term effect of growth hormone (GH) treatment on body composition in children with GH deficiency. Endocrine Journal, 46(Suppl), S35-S38.

33 Aycan, Z. \& Baş, V.N. (2014) Prader-Willi syndrome and growth hormone deficiency. Journal of Clinical Research in Pediatric Endocrinology, 6, 62-67.

34 Feigerlová, E., Diene, G., Oliver, I. et al. (2010) Elevated insulinlike growth factor-I values in children with Prader-Willi syndrome compared with growth hormone $(\mathrm{GH})$ deficiency children over two years of GH treatment. The Journal of Clinical Endocrinology \& Metabolism, 95, 4600-4608.

35 Lara-Castro, C., Luo, N., Wallace, P. et al. (2006) Adiponectin multimeric complexes and the metabolic syndrome trait cluster. Diabetes, 55, 249-259.

36 Copeland, K.C. \& Nair, K.S. (1994) Acute growth hormone effects on amino acid and lipid metabolism. The Journal of Clinical Endocrinology \& Metabolism, 78, 1040-1047.

\section{Supporting Information}

Additional supporting information may be found in the online version of this article at the publisher's web site. 